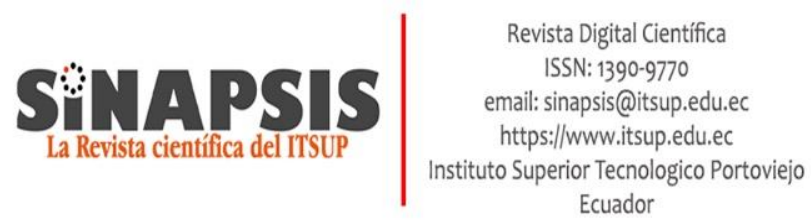

Estilo de vida y riesgo de padecer diabetes mellitus en la comunidad Joa.

Estilo de vida y riesgo de padecer diabetes mellitus en la comunidad Joa.

Delia Georgina Bravo Bonoso, Ana Parrales Choez, Solórzano Solórzano Sidar.

Revista Digital Científica, Vol. 2 Núm. 15 (2019)

@Instituto Superior Tecnológico Portoviejo, Ecuador. 


\section{Estilo de vida y riesgo de padecer diabetes mellitus en la comunidad Joa}

\section{Lifestyle and risk of diabetes mellitus in the Joa community}

Estilo de vida y riesgo de padecer diabetes mellitus

Bravo Bonoso Delia. Mg.Emg ${ }^{(1)}$

Parrales Choez Ana ${ }^{(2)}$

Solórzano Solórzano $\operatorname{Sidar}^{(3)}$

(1) Universidad Estatal del Sur de Manabí, deliabravo85@hotmail.com

(2) Universidad Estatal del Sur de Manabí,joselynparrales@hotmail.com

(3) Universidad Estatal del Sur de Manabí, sidarsolorzano@hotmail.com

Contacto:deliabravo85@hotmail.com

\section{Receptado 17/10/2019 Aceptado: 20/12/2019}

\section{Resumen}

Lo estilos de vida son todas aquellas conductas adquiridas y predominantes de una persona que determinan en gran medida su estado de salud. Un estilo de vida inadecuado es uno de los principales factores para el desarrollo de diabetes mellitus, enfermedad crónica no transmisible caracterizada por el aumento de los niveles de glucemia en la sangre, y que causa grandes afecciones en el bienestar social. Esta investigación se llevó a cabo en la comunidad de Joa del cantón Jipijapa, provincia de Manabí para determinar factores de riesgos que son predisponentes para diabetes mellitus en sus habitantes. El diseño metodológico está conformado por un estudio de tipo descriptivo de corte transversal, aplicando los métodos analítico y cuantitativo, las técnicas utilizadas fueron la entrevista a un reconocido médico diabetólogo de la provincia y la encuesta a los habitantes de la comunidad, para conocer sobre los estilos de vida y factores de riesgo, además de toma de los perímetros antropométricos, a fin de determinar el nivel de Índice de Masa Corporal y niveles de glucemia en ayunas; de 371 habitantes se utilizó la técnica de muestreo probabilístico con margen de error de 10\% dando una muestra de 58 personas, los resultados permitieron determinar que los estilos de vida en la población son inadecuados en su mayoría por lo que constituyen factores de riesgo netos que 
conjuntamente con los resultados del índice de masa corporal y glucemia indican que existe un alto riesgo de desarrollar diabetes mellitus tipo II.

Palabras clave: glucemia, bienestar social, diabetólogo.

\section{Summary}

The lifestyles are all those acquired and predominant behaviors of a person that largely determine their state of health. An inadequate lifestyle is one of the main factors for the development of diabetes mellitus, a chronic non-communicable disease characterized by the increase in blood glucose levels, and which causes major problems in social well-being. This research was carried out in the community of Joa in Jipijapa canton, province of Manabí to determine risk factors that are predisposing for diabetes mellitus in its inhabitants. The methodological design is made up of a descriptive cross-sectional study, applying the analytical and quantitative methods, the techniques used were the interview with a renowned diabetologist in the province and the survey of the inhabitants of the community, to learn about lifestyles and risk factors, in addition to taking anthropometric perimeters, to determine the level of Body Mass Index and fasting blood glucose levels; of 371 inhabitants, the probabilistic sampling technique was used with a margin of error of $10 \%$ giving a sample of 58 people, the results allowed us to determine that the lifestyles in the population are mostly inadequate and therefore constitute net risk factors that Together with the results of the body mass index and blood glucose, there is a high risk of developing type II diabetes mellitus.

Keywords: glycemia, social welfare, diabetologist.

\section{Introducción}

La Diabetes Mellitus es una enfermedad crónica no transmisible caracterizada por el incremento de los niveles glucémicos causada por la deficiente secreción de la insulina que se produce en el páncreas, o por la no asimilación de las células ante el estímulo de estas. Se relaciona comúnmente a estados de sobrepeso y obesidad, a causa del sedentarismo e inadecuados hábitos de vida (Meneses, 2014).

Según datos de la Organización Mundial de la Salud OMS la prevalencia mundial de la diabetes en adultos ha aumentado del 4,7\% en 1980 al 8,5\% en 2014, con mayor rapidez en los países de ingresos medianos y bajos, estimando que en 2015 esta enfermedad fue la causa directa de 1,6 millones de muertes, y otros 2,2 millones de muertes fueron atribuibles a la hiperglucemia, teniendo lugar antes 
de los setenta años en la mitad de los casos, y proyecta que será la séptima causa de mortalidad en 2030 (OMS, 2018).

De acuerdo a datos de la Organización Panamericana de la Salud en los países latinoamericanos y del Caribe las tasas más elevadas de prevalencia de la diabetes corresponden a Belice $(12,4 \%)$ y México (10,7\%). Managua, Ciudad de Guatemala y Bogotá mantienen tasas de alrededor del 8 al 10\% y en Ecuador se presentan índices del 5.9\% de prevalencia en la población (OPS/OMS, 2018).

En el Ecuador, según datos de la Encuesta Nacional de Salud y Nutrición (ENSANUT), en el 2013 se registró, a nivel nacional, un 62,8\% de sobrepeso y obesidad en la población entre 20 y 60 años de edad, teniendo como factores principales la mala alimentación (alto consumo calórico, bajo consumo de frutas, vegetales y fibra) y falta de actividad física (MSP, 2013). Desarrolla un porcentaje muy elevado, según cada registro de base estadístico, de poca reducción, debido a la falta de educación de estilos de vida, que no se aplican a diario (Encuesta Nacional de Salud y Nutrición, 2018).

De acuerdo a los datos estadísticos del INEC, en el año 2013 la primera causa de muerte en el Ecuador fue la diabetes mellitus donde se registraron 4.695 muertes, con una tasa de mortalidad de 29,76. Seguido tenemos enfermedades hipertensivas (tasa de mortalidad de 26,56), enfermedades cerebrovasculares y cardiovasculares (Enfermedad isquémica del corazón) con una tasa de mortalidad de 22,61 y 18,65 respectivamente, datos que pueden incrementar sus cifras en los próximos años (Instituto Nacional de Estadisticas y Censos, 2018).

De acuerdo a la Organización Panamericana de la Salud(OPS) 2016, se realizó un estudio en Ecuador, acerca de las poblaciones con riesgo de padecer enfermedades cardiovasculares, los datos obtenidos fueron abrumadores al evaluar datos recogidos de 2231 personas entre 18 y 69 años de edad, siendo un $30 \%$ de la población adulta, 40 y 69 años, susceptible de correr riesgos de padecer enfermedades cardiovasculares. Un país con alta tasa de incidencia por esta patología (OPS, 2016).

En Ecuador, de acuerdo con las cifras del Ministerio de Salud Pública, en el 2018 la prevalencia de la diabetes en adultos fue entre 20 a 79 años, es del 8.5\%, las causas que desarrollan esta enfermedad son: alimentación inadecuada, el sedentarismo, el sobrepeso, la obesidad, el consumo de tabaco y alcohol, el consumo excesivo de sal, azúcar, grasas saturadas y ácidos grasos; en el año 2014 el Instituto Nacional de Estadística y Censos (INEC) reportó como segunda causa de mortalidad general a la diabetes mellitus, situándose como la primera causa de mortalidad en la población femenina y la tercera en la población masculina (Ministerio de Salud Publica, 2018). 
Según las bases científicas de Ponce John (2015), en Manabí el número de diabéticos es de 17.985 personas, dando como resultado 11.473 mujeres y 6.512 hombres, de los cuales el 30\% de ambos grupos fueron adultos mayores, siendo Manabí la provincia más consumidora de sal, azúcar, carbohidratos, grasas, alimentos que tienden a subir de peso, y con ello favorecen el ciclo patológico de las distintas enfermedades consideradas en este grupo de pacientes.

Esto quiere decir que el 1\% de los habitantes manabitas están diagnosticados con diabetes, y un gran porcentaje están en riesgo de padecerla, situación promovida por los patrones conductuales presentes en la provincia manabita, ya que se suele mantener una dieta hipocalórica, alta en grasas y carbohidratos sumado a la poca actividad física que se realiza y a las condiciones genéticas, por lo que el riesgo de diabetes en los manabitas es alto.

Entre los factores patogénicos puede englobarse variables genéticas, ambientales e inmunológicas, las cuales ocasionan diversos efectos adversos en el organismo, como la destrucción de las células $\beta$ del páncreas, lo que conlleva a disminuir la producción de insulina, hormona encargada de regular los niveles de glucosa en la sangre, o también provocar que la insulina no surja efecto en el organismo. En cualquiera de los dos casos un incremento de glucosa en la sangre promueve el riesgo de desarrollar episodios cardiovasculares (Funes, Diabetes y trabajo. Análisis de la influencia del control de la enfermedad y de las condiciones de trabajo en el absentismo laboral de las personas diabéticas, 2012).

El incremento de los niveles de azúcar o glucosa en la sangre se la conoce como hiperglicemia, esta provoca un daño prolongado, limitando las funciones de los órganos como los ojos, riñones, nervios, corazón y vasos sanguíneos (Rizo \& Sandoval, Comportamiento clínico - epidemiológico de la diabetes mellitus en niños y adolescentes atendidos en consulta externa, Hospital Manuel de Jesús rivera "La Mascota" durante Enero 2012 - Junio 2014, 2016). Este acelerado y progresivo daño disminuye la calidad de vida de los pacientes que la padecen, y esta multi afectación provoca el desarrollo de complicaciones crónicas que conlleva a la invalidez y a la muerte prematura (Domínguez, Licea, y (Domínguez, Licea, \& Hernández, 2018).

En este sentido Quisiguiña (2010), informa que por la alta prevalencia de esta enfermedad, promovida por los diversos factores, tanto genéticos como ambientales, se considera un problema importante de salud pública, por lo que los esfuerzos que se realizan están enfocados a las estrategias preventivas mediante el mejoramiento de los hábitos de vida, que condicionan en gran magnitud el de sarrollo o 
no de esta condición, por lo tanto resulta imprescindible conocer el temprano diagnóstico y reconocimiento de los factores de riesgo de esta enfermedad.

La Diabetes Mellitus en sus primeras etapas no suele presentar sintomatología, por lo que a veces se ignora su estado, si es que el paciente no se realiza los chequeos médicos establecidos en los programas del MSP, el diagnóstico se realiza años después, cuando ya se manifiestan las primeras complicaciones, por tal razón es considerada una enfermedad silenciosa que poco a poco va degenerando las funciones del organismo, ocasionando el deterioro progresivo del estado de salud del que lo padece (Barbecho y Ortiz 2016).

Por consiguiente, no existe un patrón definitivo en cuanto al surgimiento de las complicaciones, pero si se puede definir los efectos de un inadecuado control o descuido de la enfermedad. Cuando un diabético no lleva un control de su condición puede surgir la descompensación hiperglucémica cetoacidótica y la descompensación hiperglucémica hiperosmolar. Estas suelen ser las condiciones de mayor causa de los ingresos hospitalarios en pacientes diabéticos (Pérez, 2015).

La Diabetes Mellitus tiene su clasificación primaria de dos tipos: la de Tipo I se produce cuando el páncreas deja de producir insulina o esta se destruye, un subtipo es debido a un desequilibrio o trastorno autoinmune, aunque en un porcentaje de pacientes no haya evidencia de esta causa, en otro subtipo, la idiopática, la causa principal se ha determinado por ser hereditaria. Su efecto es ocasionar la hiperglicemia, que puede presentarse en edades tempranas juveniles y es muy probable que la aparición no tome tiempo en diagnosticarla, este tipo afecta a cerca del 10\% de los diabéticos, por lo que suele ser la menos común (Ayte, 2018).

Cuando un paciente diabético, ante un inadecuado control, manifiesta una crisis hiperglicemia, la cual muestra síntomas como deshidratación moderada a severa, perdida sensorial, aumento de la frecuencia respiratoria que puede ser de tipo Kussmal, es decir rápida y profunda, vómitos y dolor abdominal (Garcia, 2018).

El desarrollo del conocimiento del comportamiento de esta enfermedad se ha venido acrecentando en las últimas décadas, es así que contrario a lo que antes se sostenía respecto a que la diabetes incurre solamente en una elevación de los niveles de glucosa en la sangre, en los actuales tiempos se conoce que esta enfermedad está determinada por una serie de causas fenotípicas basadas en variables genéticas, ambientales e inmunitarias, significando diferencias marcadas en cuando a las causas, complicaciones y reacción del organismo ante el tratamiento de la enfermedad. 
Para esto es necesario que el tratamiento médico se aplique desde diversos criterios según la complicación, ya que, al presentarse en diversas manifestaciones clínicas, con diversas causantes y formas de presentación, resulta muy probable que no se pueda atender todas las variables que conjugan la complicación, y con esto se puede promover un deterioro en un órgano que no se esté tratando (Reyes, Pérez, Figueredo, Ramírez, \& Jiménez, 2016).

En una enfermedad como la diabetes es importante como medida general, establecer un cambio en el patrón conductual, que a su vez se direccione en mejorar el estado de salud y mitigar complicaciones propias de la enfermedad, que pongan en riesgo la vida de la persona. En este sentido el estilo de vida cambia drásticamente poniendo limitaciones en su diario vivir, modificando desde su ingesta hasta sus actividades que influyan en el proceso metabólico.

Este cambio, supone en el paciente diabético, diversas reacciones, desde el rechazo y resistencia a los nuevos cambios, como estados depresivos y desarrollo de problemas psicológicos, hasta la aceptación y puesta en práctica de las medidas necesarias para establecer el mejoramiento en la salud. Es importante, por lo consiguiente, que estos cambios estén acompañados por la asistencia profesional para direccionar el cambio en el estilo de vida hacia una conducta que coadyuve al bienestar de la persona.

Todo lo ante expuesto y según diversas investigaciones afirman que esta enfermedad es incurable, pero si tratable una vez diagnosticada, pero las personas que la padecen tienen la oportunidad de estabilizar su calidad de vida si cumple con las orientaciones médicas al respecto. Actualmente se han desarrollado grandes avances en el tratamiento de la enfermedad, por supuesto, esto supone que el paciente cumpla con todas las medidas de cuidado planteadas por el médico, cosa que en la práctica a muchas personas les resulta difícil, ya que muy a menudo tiene que modificar totalmente sus estilos de vida, no todos reaccionan con la misma predisposición. Una situación óptima sería el constante tratamiento farmacológico, un adecuado estilo de vida, y medición constante de la glicemia, así se logra minimizar las complicaciones y se extiende la vida del paciente (Quílez y García, 2015).

El impacto social de esta enfermedad alcanza grandes magnitudes, debido a su alta morbimortalidad, tornándose dependiente tanto del tratamiento como de la atención de sus complicaciones, que se derivan de esta. Un gran porcentaje de personas diabéticas, desarrollan enfermedades cardiovasculares, esta última suele ser la causa principal de mortalidad en diabéticos, incluso en mayor proporción comparado con la población general. Es tan alto el riesgo de enfermedades 
cardiovasculares en la diabetes que a menudo es tomada como una enfermedad coronaria, aunque en realidad se trate de un factor de riesgo, y por lo tanto se pueda controlar.

Por tanto, es indispensable determinar el riesgo de padecer un episodio cardiovascular en un paciente diabético, ya que frecuentemente se asocia a otros factores de riesgo aterogénicos. Al conocer el índice de riesgo se contribuye a la disminución de estos episodios, siendo una herramienta eficaz para este propósito (Oliveira, Costa, Ferreira, y Lima 2017).

Las medidas preventivas para evitar en enfermedades cardiovasculares están basadas en lineamientos primarios y secundarios. El componente primario se basa en conocer los índices de presencia en la población, identificar a los pacientes que la padecen para que se tomen las acciones necesarias en el inmediato cuidado y así evitar o aplazar las situaciones de crisis cardiovasculares. En cuanto al componente secundario está determinada por el tratamiento y la rehabilitación cardiaca una vez ya hayan aparecido los síntomas, teniendo como propósito la recuperación del paciente y el cuidado para evitar o mitigar los futuros episodios cardiovasculares (Vega, y otros, 2018).

Desde estos antecedentes surge expectativa en el presente trabajo de investigación científica, ya que a través del conocimiento empírico se puede evidenciar que los usuarios a pesar de estar informados acerca de esta patología y el buen estilo de vida incurren en los malos hábitos; En este contexto en la comunidad de Joa, se evidencia que existe un insuficiente conocimiento sobre esta patología y sus complicaciones, lo cual les lleva a desencadenar la enfermedad, entre las causas más comunes para la prevalencia de esta enfermedad pudieran considerase: el consumo de alimentos con alto contenido de grasa, la obesidad, el sedentarismo, la falta de ejercicio físico, el alcoholismo y el tabaquismo, lo cual demuestra que son insuficiente los conocimientos del autocuidado de los habitantes.

Este acercamiento teórico de la problemática estudiada permitió determinar como objeto de investigación, los estilos de vida y los riesgos de desarrollar Diabetes Mellitus tipo II. El objetivo general es determinar estilos de vida predisponente para diabetes mellitus en los habitantes de la comunidad de Joa. Del principal objetivo se desglosan las siguientes Objetivos específicos: Identificar factores de riesgos predisponentes para el desarrollo de diabetes mellitus teniendo como punto de partida el diagnostico de salud existente; Valorar índice de masa corporal y resultados glicémicos en la población con factores de riesgos predisponente de diabetes en la comunidad de Joa; Establecer patrones saludables de vida en la comunidad de Joa, enfatizando en los esquemas de alimentación. 
Se justifica el sustento de esta investigación y su relevancia en conocer los riesgos predisponentes de desarrollar Diabetes Mellitus tipo II. Por estas razones el presente trabajo de investigación tiene como finalidad, conocer los estilos de vida y los posibles riesgos de desarrollar diabetes mellitus en la comunidad de Joa, de esta manera contribuir con los habitantes, a fin de mejorar su calidad de vida y reconocer los riesgos de diabetes evitando su desarrollo y por ende sus complicaciones, las técnicas que se utilizarán para la elaboración y desarrollo de la investigación serán las encuestas aplicada a los usuarios de esta comuna.

\section{Materiales y Métodos}

La presente investigación se realizó mediante la aplicación del método descriptivo, que a su vez nos permitió describir con fundamentos teóricos sobre los diferentes estilos de vida, que juegan un papel importante en cuanto al riesgo predisponente de padecer diabetes mellitus, fue de cohorte transversal, ya que se recolectaron los datos en un tiempo determinado. Se aplicó también el método analítico que permitió conocer a profundidad el objeto de estudio y con el método cuantitativo se logró recopilar datos cuantificables y en base a esto se realizaron los análisis estadísticos.

Se utilizaron las técnicas e instrumentos como herramientas para la recolección de datos: la encuesta misma que se llevó a cabo en la comunidad de Joa, el formulario aplicado estuvo estructurado con un encabezado de los datos sociodemográficos de los habitantes, más 10 preguntas sencillas con respuestas directas, las cuales se diseñaron en base a los objetivos específicos, con el fin de identificar los riesgos que conllevan a desarrollar la Diabetes Mellitus tipo II, información necesaria para la investigación, logrando obtener datos y resultados reales y confiables para el estudio.

Los datos obtenidos se recopilaron, procesaron y tabularon a través de software estadístico Ms Excel 2019, procediendo a la interpretación y análisis de los resultados. Se justifica el sustento de esta investigación y su relevancia en conocer los riesgos predisponentes de desarrollar Diabetes Mellitus tipo II. Por estas razones el presente trabajo de investigación tiene como finalidad, conocer los estilos de vida y los posibles riesgos de desarrollar diabetes mellitus en la comunidad de Joa, de esta manera contribuir con los habitantes, a fin de mejorar su calidad de vida y reconocer los riesgos de diabetes evitando su desarrollo y por ende sus complicaciones, las técnicas que se utilizarán para la elaboración y desarrollo de la investigación serán las encuestas aplicada a los usuarios de esta comuna.

El presente proyecto de investigación se llevó a cabo en el sector rural Joa, que está ubicado a $5 \mathrm{~km}$ al oeste del cantón Jipijapa, tomando en dirección a Puerto Cayo, este sector está ubicado al sur de la provincia de Manabí, 
Se realizó un estudio censal, descriptivo, de cohorte transversal con el método analítico y cuantitativo mediante las variables: estilo de vida (independiente) y riesgo de padecer diabetes mellitus (dependiente).

Para la obtención de los resultados se utilizaron 2 técnicas de investigación: la entrevista y la encuesta, la primera aplicada al Dr. Jonathan Villacís médico diabetólogo, encargado del área de diabetes en el Hospital Rafael Rodríguez Zambrano de la ciudad de Manta, y la segunda aplicada a los habitantes de la comunidad Joa del cantón Jipijapa, que es la zona de estudio, la población fue de 371 habitantes con una muestra probabilística de 58 personas de entre 20 a 80 años que tienen riesgo de padecer diabetes.

\section{Discusión}

En la Diabetes Mellitus de tipo II, el organismo la insulina producida es muy defectuosa o no alcanza los niveles necesarios para sus funciones metabólicas, además los tejidos ofrecen una gran resistencia a asimilarla, suele aparecer cuando el individuo ya tiene una edad madura, aunque esta condición no es del todo particular (Alonso, 2015).

En cuanto al sexo, el 62\% de los encuestados son de sexo femenino y el $38 \%$ son de sexo masculino. Existe una diferencia significativa entre ambos sexos, se estima que $2 / 3$ a favor de las mujeres en esta investigación. En cuanto a su ocupación el 52\% tiene de ocupación ser ama de casa y el 34\% se dedica a la agricultura, lo que a su vez genera un desconocimiento sobre esta patología.

Aucay \& Carabajo señalan resultados similares, indican en su investigación que la presencia de Diabetes Mellitus tipo II, fue más frecuente en el género femenino con un porcentaje $61 \%$, y en las personas mayores de 60 años con 57.9\%, al igual que Reyes, la cual indica en los resultados de su estudio, que el 75\% de los diabéticos son del género femenino, los resultados de los habitantes de Joa coinciden con los autores antes mencionados, demostrando que el género femenino tuvo más prevalencia de riesgos de padecer diabetes mellitus II (Aucay \& Carabajo, 2013) (Reyes C. , 2012).

La asociación estadísticamente significante encontrada entre factores de riesgo para DM2 y áreas del conocimiento no puede ser discutida debido a la falta de literatura, lo que hace esa relación todavía desconocida, representando un reto para futuras investigaciones. Así, futuramente, es importante más allá de los datos cuantitativos, conocer los matices, relacionados al género y grupo de edad de esta camada social, que los impiden de seguir una dieta adecuada y practicar ejercicios físicos regulares. 
Por tanto, diagnosticar no es sinónimo de prevenir si no se dispone de una estrategia de intervención adecuada. La primera posibilidad real de prevenir la diabetes y sus complicaciones es intervenir sobre el conjunto de factores de riesgo a través de la modificación de los estilos de vida. Diversos estudios ya han demostrado la eficacia de estas intervenciones para reducir la incidencia de diabetes en condiciones bien estructuradas de aplicación. (Sanso \& Alonso , 2010).

\section{Resultados.}

De la población de estudio, el 95\% tiene valor glucémico mayor que $100 \mathrm{mg} / \mathrm{dl}$ a $126 \mathrm{mg} / \mathrm{dl}$, datos mostrados en la tabla 1, valores considerados de riesgo para padecer diabetes, cifras que alarman a los entes de salud ya que, a su vez, se vuelve una problemática de carácter social, económico y demográfico.

El segundo componente es la encuesta aplicada para recabar información sobre los estilos de vida de la población y los factores de riesgo asociados con el desarrollo de la diabetes mellitus, con lo cual se determinó que:

El 100\% de los encuestados manifestó, que en sus familiares existe un integrante con diabetes, por lo que esto representa un riesgo genético para padecer esta enfermedad. Y en relación a otras enfermedades se evidencia que existe alto índice de hipertensión, el a su vez uno de sus familiares padecen de insuficiencia renal. Por tanto, en los encuestados se determinan afecciones que se asocian como factores de riesgos para padecer diabetes mellitus.

\begin{tabular}{|c|c|c|}
\hline Alternativas & Frecuencia & Porcentajes \\
\hline$<100$ & 3 & $5 \%$ \\
\hline 100 a 110 & 21 & $36 \%$ \\
\hline 111 a 120 & 21 & $36 \%$ \\
\hline 121 a 126 & 13 & $23 \%$ \\
\hline Total & 58 & $100 \%$ \\
\hline
\end{tabular}

Tabla 1. Glucemia

Los estilos de vida están marcados por las conductas referentes a la actividad física, y la ingesta de alimentos, así el $62 \%$ de los encuestados afirman, que no realizan actividad física, de estos, el $41 \%$ solo realizan actividades de 3 a 5 veces a la semana y el 36\% manifiesta que nunca, tal como se 
muestra en la tabla 2; 30 minutos diarios, es una frecuencia recomendable, solo 1/4 de la población cumple, se confirma el porcentaje de personas que no realizan ningún tipo de actividad física que es más $1 / 3$ de los encuestados. Este alto porcentaje se relaciona directamente con el riesgo de padecer diabetes mellitus.

\begin{tabular}{|c|c|c|}
\hline Alternativas & Frecuencia & Porcentajes \\
\hline 30 minutos diarios & 13 & $23 \%$ \\
\hline 3 a 5 veces a la semana & 24 & $41 \%$ \\
\hline Nunca & 21 & $36 \%$ \\
\hline Total & 58 & $100 \%$ \\
\hline
\end{tabular}

Tabla 2. Frecuencia de actividad física

Esto concuerda con los resultados de Moposita, quien en su investigación determinó que su población de estudio realiza actividad física de leve intensidad, muy pocos pacientes realizan actividad intensa, además que la alimentación es de regular a mala, lo que también concuerda con las siguientes interrogantes de este estudio (Moposita, 2012).

La ingesta alimenticia también predispone riesgo de desarrollar diabetes, en el estudio se comprobó que el $43 \%$ consume en su mayoría carne, el 33\% grasas y el 7\% en su dieta más consume frituras, señalados en la tabla 3. A pesar de que la alimentación tiene que ser equilibrada en todos sus aportes nutricionales, se percibe que 4/10 encuestados consumen grasas y frituras en mayor proporción que el resto de alimentos.

\begin{tabular}{|c|c|c|}
\hline Alternativas & Frecuencia & Porcentajes \\
\hline Frutas y verduras & 10 & $17 \%$ \\
\hline Carnes & 25 & $43 \%$ \\
\hline Grasas & 19 & $33 \%$ \\
\hline Frituras & 4 & $7 \%$ \\
\hline Total & 58 & $100 \%$
\end{tabular}

Tabla 3. Alimentos de mayor consumo

En cuanto a la frecuencia con que se alimentan diariamente los habitantes de Joa se destaca que, el $62 \%$ de ellos comen de 3 a 4 veces al día y el $26 \%$ lo hace de 4 a 5 veces al día. De acuerdo a la teoría 
nutricional, se debe alimentar 5 veces al día en porciones moderadas, lo que manifiesta una gran parte de la población, sin embargo, se desconoce si las porciones que consumen son las adecuadas y la nutrición es balanceada.

Resultados similares exponen Chisaguano \& Ushiña, quienes refieren que el estilo de vida de los pacientes diabéticos en su población de estudio no es adecuado debido a que el 30\% consume pan, se agrega sal a la dieta en un 30\% y el consumo de azúcar en el 20\% se lo consume de forma diaria (Chisaguano \& Ushiña, 2015).

Los hábitos de consumo no saludables también coadyuvan a desarrollar diabetes mellitus, así el 84\% de los encuestados manifiestan que sí tiene algún hábito de consumo no saludable conocido por afectar a la salud, entre los que están el consumo de alcohol en el $28 \%$ y de café con el $38 \%$, como se detalla en la tabla 4. El alto índice de personas que reconocen mantener un hábito de consumo no saludable, conlleva a que exista una relación entre estos y la presencia de diabetes mellitus.

\begin{tabular}{|c|c|c|}
\hline Alternativas & Frecuencia & Porcentajes \\
\hline Cigarro & 6 & $10 \%$ \\
\hline Tabaco & 9 & $15 \%$ \\
\hline Alcohol & 16 & $28 \%$ \\
\hline Café & 22 & $38 \%$ \\
\hline Ninguno & 5 & $9 \%$ \\
\hline Total & 58 & $100 \%$ \\
\hline
\end{tabular}

Tabla 4. Tipos de hábitos de consumo no saludable

De forma general Figueroa contrasta estos resultados sobre los factores de riesgo, quien señala en su investigación, que estos primordialmente se dan por el nivel socioeconómico bajo, mal control metabólico y tabaquismo (Figueroa, 2019). De los encuestados afirma consumir algún tipo de medicamento, entre los que están para el tratamiento de hipertensión, analgésicos y AINES.

Finalmente, el 76\% manifiesta no tener conocimiento sobre el factor de riesgo de sobrepeso en el desarrollo de enfermedades como la diabetes. Es notable el alto nivel de desconocimiento de los habitantes de la comunidad de Joa. 


\section{Conclusiones}

Se concluye en que los factores de riesgo predominantes para el desarrollo de diabetes mellitus en la comunidad de Joa, son el sobrepeso y la obesidad, partiendo de un diagnóstico previo, esto inclina a los habitantes de dicha comunidad, a modificar los estilos de vida que conllevan a esta afección, por lo que, ante lo expuesto esta patología se convierte en una de las enfermedades más prevalentes en la Zona Sur de Manabí.

En la comunidad de Joa del cantón Jipijapa se pudo determinar, que en la población de estudio existe un alto riesgo de contraer diabetes, ya que todos presentaron índices de sobrepeso y obesidad que oscilan entre 25.0 a 29.9, y en su mayoría valores glucémicos en ayunas con valores de 100 a 126 $\mathrm{Mg} / \mathrm{dl}$, que son indicadores de riesgo para desarrollar Diabetes Mellitus II.

Se pudo establecer, que los estilos de vida saludables son escasos en esta localidad, lo que permitió a su vez determinar los riesgos que predisponen a desarrollar esta patología, la mayor parte de los habitantes no realizan actividad física, llevan una dieta calórica inadecuada y abusan del consumo de productos alimenticios no saludables, por tanto, los riesgos de desarrollar la diabetes mellitus tipo II en la comunidad de Joa es muy alto.

\section{Bibliografía}

OPS. (2018).La diabetes en las personas mayores. Recuperado de http://www.helpagela.org/silo/files/factsheet-diabetes.pdf

Acosta, I. (2013). Cuidados de enfermería a pacientes hospitalizados con pie diabético en el Hospital Guayaquil Dr. Abel Gilbert Ponton del Ministerio de Salud Pública. Tesis de grado, Universidad Católica Santiago de Guayaquil, Facultad de Ciencias Medicas, Guayaquil, Ecuador.

Acuña, Y., \& Cortes, R. (2014). Promoción de estilos de vida saludable Área de Salud de Esparza. Tesis de maestría, Instituto Centroamericano de administración Pública, Costa Rica.

Alonso. (2015). Guías Clínicas. Diabetes Mellitus. Badalona: Euromedice. Ediciones Médicas, S.L. Anchante, M. (2018). Estilos de vida saludable y estrés laboral en enfermeras de áreas críticas del Instituto Nacional de Salud del Niño, Lima, 2017. Tesis de maestría, Universidad César Vallejo, Escuela de Posgrado, Lima.

Aucay, O., y Carabajo, L. (2013). Autocuidado en personas con diabetes mellitus tipo 2, que asisten al club de diabéticos del Centro de Salud $N^{\circ} 1$ Pumapungo, Cuenca, Ecuador 2013. Universidad de Cuenca, Facultad de Ciencias Médicas, Cuenca, Ecuador. 
Ayte, V. (2018). Estilos de vida en usuarios con diabetes mellitus tipo II de los consultores externos del Hospital San Juan de Lurigancho, 2018. Tesis de grado, Universidad Norbert Wiener, Facultad de Ciencias de la Salud, Lima.

Bacusoy, F. (2019). Impacto del programa público en el control de diabetes mellitus 2 en beneficiarios. Proyecto de investigación, Universidad Estatal Del Sur De Manabí, Facultad Ciencias de la Salud, Jipijapa, Ecuador.

Barahona, M. (2019). Estrategias de autocuidado y prevención de complicaciones en pacientes diabéticos. Proyecto de investigación, Universidad Estatal del Sur de Manabí, Facultad de Ciencias de la Salud, Jipijapa, Ecuador.

Barbecho, J., y Ortiz, L. (2016). Uso de los estándares de cuidado médico de la asociación americana de diabetes 2014, para el control metabólico de diabetes mellitus tipo 2, a ser realizado en el centro de salud $n^{\circ} 2$ Las Casas del distrito 17D05, durante el 2015. Tesis de Posgrado, Pontificia Universidad Católica del Ecuador, Facultad de Medicina. Posgrado de Medicina Familiar y Comunitaria, Quito.

Canova-Barrios, C. (2017). Estilo de vida de estudiantes universitarios de enfermería de Santa Marta, Colombia. Rev Colomb Enferm, 14(23-32).

Chisaguano, E., \& Ushiña, R. (2015). Estilos de vida en los pacientes con diabetes tipo II que asisten al club de diabeticos del Centro de Salud N.-1 de la ciudad de Latacunga de julio - agosto del 2015. Universidad Central del Ecuador, Facultad de Ciencias Médicas, Quito.

Chun, S. (2017). Prácticas de estilo de vida saludable en el personal de enfermería, distrito de salud Santa Lucía La Reforma. Tesis de grado, Universidad Rafael Landívar, Facultad de Ciencias de la Salud, Guatemala.

Domínguez, Y., Licea, M., \& Hernández, J. (octubre de 2018). Algunos apuntes sobre la Epidemiología de la diabetes mellitus tipo 1. Revista Cubana de Salud Pública, 44(3).

Encuesta Nacional de Salud y Nutrición. (2018). Recuperado el Agosto de 2019, de ENSANUT.

Fernández, A. R., \& Manrique-Abril, F. G. (Agosto de 2010). Efecto de la intervención educativa en la agencia de autocuidado del adulto mayor hipertenso de Boyacá, Colombia. Ciencia y enfermería, 16(2).

Figueroa, B. (2019). Factores de riesgo en adultos con Pie diabético de 41 a 60 años de edad. Universidad Estatal del Sur de Manabí, Facultad de Ciencias de la Salud, Jipijapa, Ecuador. Fortea, M. (2017). Impacto de un programa educativo en el control de la diabetes mellitus tipo 2. Tesis doctoral, Universitat Jaume I, Castellón. 
Funes, J. (2012). Diabetes y trabajo. Análisis de la influencia del control de la enfermedad y de las condiciones de trabajo en el absentismo laboral de las personas diabéticas. Tesis doctoral, Universitat Autònoma de Barcelona, Department de Pediatria, d' Obstetricia i ginecología i de Medicina Preventiva, Bellaterra.

Funes, J. (2012). Diabetes y trabajo. Análisis de la influencia del control de la enfermedad y de las condiciones de trabajo en el absentismo laboral de las personas diabéticas. Tesis doctoral, Universitat Autonoma de Barcelona, Departament de Pediatria, d’Obstetrícia i Ginecologia i de Medicina Preventiva, Barcelona.

Garcia, C. (2018). Asociación entre la depresión y las complicaciones tardías de la diabetes mellitus tipo 2 en el Hospital Nacional Luis N. Sáenz PNP. Tesis de grado, Universidad Ricardo Palma, Facultad de Medicina Humana, Lima.

García, D. (2019). Estilo de vida y complicaciones en diabéticos tipo II mayores de 30 años. Proyecto de investigación, Universidad Estatal del Sur de Manabí, Facultad de Ciencias de la Salud, Jipijapa, Ecuador.

García, M., \& Durruty, P. (2009). Prevención de la Diabetes Mellitus tipo 2. Rev Med Clin Condes, 20(5), 580-587.

Guerrero, J. (2014). Caracterización de los estilos de vida de los escolares de las escuelas básicas nacionales de la parroquia Juan de villegas como factores de riesgo para el desarrollo de enfermedades crónicas. Barquisimeto: Universidad Centrooccidental "Lisandro Alvarado".

Gutiérrez, S. (2015). Características Clínico-Epidemiológicas de la Cetoacidosis Diabética en Pacientes Pediátricos que asisten al Hospital Dr. Mario Catarino Rivas. Posgrado de Pediatría, Universidad Nacional Autónoma de Honduras, Escuela Universitaria de Medicina, Honduras.

HBSC. (2015). Estilos de vida y Promoción de la Salud. American Journal of Public Health.

Instituto Nacional de Estadisticas y Censos. (2018). Recuperado el Agosto de 2019, de INEC.

Jirón, J., \& Palomares, G. (2018). Estilos de vida y grado de dependencia en el adulto mayor en el Centro de Atención Residencial Geronto Geriátrico Ignacia Rodulfo Viuda de Canevaro, Rimac - 2018. Tesis de grado, Universidad Norvert Wiener, Facultad de Ciencias de la Salud, Lima.

Llorente, Y., Miguel-Soca, P., Rivas, D., \& Borrego, Y. (2016). Factores de riesgo asociados con la aparición de diabetes mellitus tipo 2 en personas adultas. Revista Cubana de Endocrinología, 27(2), 123-133.

Loría, T. (2014). Caracterización de los estilos de vida, nivel de actividad física y prevalencia de obesidad en la población estudiantil de la sede de occidente de la Universidad de Costa Rica: Aporte para un plan de intervención. Tesis de maestría, Universidad Nacional, Sistema de Estudio de Posgrado, Costa Rica. 
Macías, K. (2018). Factores de riesgo asociados a diabetes por embarazo en pacientes atendidas en Centro de Salud Jipijapa. Proyecto de investigación, Universidad Estatal del Sur de Manabí, Facultad Ciencias de la Salud, Jipijapa, Ecuador.

Martínez, M. (2013). Utilidad de las encuestas de salud para la investigaciónepidemiológica de la diabetes en España. Tesis doctoral, Universidad Complutense de Madrid, Facultad de Medicina, Madrid.

Meneses, C. (2014). Conocimientos que tienen los pacientes diabéticos sobre diabetes mellitus tipo 2 en una unidad de medicina familiar. Tesis De Posgrado, Universidad Veracruzana, Córdoba Veracruz.

Ministerio de Salud Publica. (2018). Recuperado el 2018, de guias de practicas clinicas: https://www.salud.gob.ec/wp-content/uploads/downloads/2017/05/Diabetesmellitus_GPC.pdf

Moposita, A. (2012). Factores en la adhesión al tratamiento farmacológico y no farmacologico en pacientes con diagnóstico de diabetes mellitus tipo 2 . Ambato, Ecuador: Universidad Regional Autónoma de Los Andes.

Oliveira, P., Costa, M., Ferreira, J., \& Lima, C. (enero de 2017). Autocuidado en Diabetes Mellitus: estudio bibliométrico. Enfermería global, 45, 634 - 652.

OPS/OMS. (2018). Recuperado el Agosto de 2019, de Diabetes. : https://www.paho.org/hq/index.php?option=com_content\&view=category\&id=4475\&layout $=$ blog\&Itemid=40610\&lang=es\&limitstart $=15$

Organizacion Panamericana de la Salud (OPS). (2016). Recuperado el Julio de 2019

Palacios, A., Durán, M., \& Obregón, O. (2012). Factores de riesgo para el desarrollo de diabetes tipo 2 y sindrome metabólico. Revista Venezolana de Endocrinología y Metabolismo, 10(1), 34-40.

Pérez, R. (2015). Influencia de la diabetes Mellitus tipo 1 y su control metabólico sobre la proporción de células de extirpe osteobástica en sangre periférica en niños y adultos jovenes. Tesis doctoral, Universidad de Sevilla, Sevilla.

Pincay, M. (2019). Intervención de enfermería en el autocuidado en pacientes diabeticos tipo II atendidos en el primer nivel de salud. Proyecto de investigación, Universidad Estatal del Sur de Manabí, Facultad de Ciencias de la Salud, Jipijapa, Ecuador.

Ponce, J. (2015). Descripción de los estilos de vida asociados a Diabetes Mellitus Tipo II. tesis, Universidad San Francisco, Quito.

Ponte, M. (2013). Estilo de vida de los estudiantes de enfermería, según año de estudio de la Universidad Nacional Mayor de San Marcos. Tesis de grado, Universidad Nacional Mayor de San Marcos, Facultad de Medicina Humana, Lima. 
Quílez, P., \& García, M. (diciembre de 2015). Control glucémico a través del ejercicio físico en pacientes con diabetes mellitus tipo 2; revisión sistemática. Nutrición hospitalaria, 31(4), 14651472.

Quintero, J. (2014). Hábitos alimentarios, estilos de vida saludables y actividad física en neojaverianos del program nutricióny dietética de la PUJ Primer periodo 2014. Tesis de grado, Pontificia Universidad Javeriana, Facultad de Ciencias, Bogotá.

Quisiguiña, D. (2010). Factores que inciden en el incrementeo de las complicaciones crónicas de la diabetes mellitus tipo 2 en el servicio de medicina interna del Hospital Alfredo Noboa Montenegro. Tesis de grado, Escuela Superior Politécnica de Chimborazo, Facultad de Salud Pública. Escuela de Medicina, Riobamba.

Reyes, C. (2012). Autocuidado y facotres de riesgo en pacientes diabéticos que acuden a la consulta externa del cantro de salud KJipijapa del cantón Jipijapa en el periodo Febrero - Julio 2012. Tesis de grado, Universidad Estatal del Sur de Manabí, Unidad Académica de Ciencias de la Salud, Jipijapa, Ecuador.

Reyes, F., Pérez, M., Figueredo, E., Ramírez, M., \& Jiménez, Y. (2016). Tratamiento actual de la diabetes mellitus tipo 2. Correo Científico Médico de Holguín, 20(1).

Rizo, M., \& Sandoval, K. (2016). Comportamiento clínico - epidemiológico de la diabetes mellitus en niños y adolescentes atendidos en consulta externa, Hospital Manuel de Jesús rivera "La Mascota" durante Enero 2012 - Junio 2014. Tesis de investigación, Universidad Nacional Autónoma de Nicaragua, Facultad de Ciencias Médicas, Managua.

Rizo, M., \& Sandoval, K. (2016). Comportamiento clínico-epidemiológico de la diabetes mellitus en niños y adolescentes atendidos en consulta externa Hospital Manuel de Jesús Rivera. Tesis de investigación, Universidad Nacional Autónoma de Nicaragua, Facultad de Ciencias Médicas, Guatemala.

Salud, M. d. (2014). Guía Práctica Clínica Nacional sobre Prevención, Diagnóstico y Tratamiento de la Diabetes Mellitus Tipo 2. Ministerio de Salud. Presidencia de la Nación. Argentina, Buenos Aires.

Sanabria, P., González, L., \& Urrego, D. (2014). Estilos devida saludables en profesionales de la salud colombianos, estudio ecploratorio. Universidad Militar Nueva Granada, Facultad de Medicina, Bogotá, Colombia.

Sánchez, B., Bonilla, J., Peña, E., Costa, M., \& Zerquera, G. (2016). Percepción de riesgo de desarrollar diabetes mellitus en personas no diabeticas. Revista Finlay, 6(2). 
Sánchez, E. (2016). Estilos de vida y salud en el elumnado de la facultad de Ciencias de la Educación de la Universidad de Málaga. Tesis doctoral, Universidad de Málaga, Facultad de Ciencias de la Salud, Málaga.

Sánchez, L. (2019). Autocuidado y calidad de vida en pacientes con diabetes mellitus tipo II. Tesis de grado, Universidad Estatal del Sur de Manabí, Facultad Ciencias de la Salud, Jipijapa, Manabí.

Sanso, F., \& Alonso , P. (2010). Guia terapeutica para la Atencion Primaria de Salud. La Habana: ECIMED.

Simó, R., \& Hernández, C. (2012). Tratamiento de la diabetes mellitus: objetivos generales y manejo de la práctica clínica. Rev Esp Cardiol, 55(8).

Soler, Y., Pérez, E., López, M., \& Quezada, D. (2016). Conocimientos y autocuidado en pacientes con diabetes mellitus. Rev. Arch Med Camagüey, 20(3).

SSAE- ASSE- MSP, U. I. (2012). Diabetes Mellitus Tipo 2. guía de intervenciones en el primer nivel de atención. MSP, Escuela Universitaria de Tecnología Médica.

Tobón Correa, O. (s.f.). El Autocuidado, una habilidad para vivir. Recuperado el Agosto de 2019, de El autocuidado, una habilidad vara http://www.alcoholinformate.com.mx/AUTOCUIDADO.pdf

Vega, J., Verano, N., Rodríguez, J., Labrada, E., Sánchez, A., \& Espinosa, L. (2018). Factores cardioaterogénicos y riesgo cardiovascular en diabéticos tipo 2 hospitalizados. Revista Cubana de Medicina Militar, 47(2).

Véliz, T. (2017). Estilos de vida y salud: Estudio del caso de los estudiantes de la Facultad de ciencias médicas, Universidad de San Carlos de Guatemala. Tesis doctoral, Universitat de Barcelona, Programa de doctorado en sociología, Barcelona.

Xandri, R. (2017). Estilos de vida, personalidad y emocionalidad negativa en estudiantes universitarios. Tesis doctoral, Universidad Católica de Murcia, Escuela Internacional de Doctorado, Murcia. 
